\title{
Criminologie
}

\section{Enjeux du travail policier en milieux autochtones au Québec} La méthode d'analyse en groupe au service de la recherche de solutions

\section{Policing issues in Indigenous communities of Quebec} The group analysis method in the search for solutions

\section{Asuntos del trabajo policial en las comunidades indígenas en Quebec}

\author{
El método de análisis de grupo al servicio de la búsqueda de \\ soluciones
}

\author{
Annie Gendron, Nina Admo, Chantal Plourde et Isabelle Thibault
}

Volume 53, numéro 2, automne 2020

URI : https://id.erudit.org/iderudit/1074198ar

DOI : https://doi.org/10.7202/1074198ar

Aller au sommaire du numéro

Éditeur(s)

Les Presses de l’Université de Montréal

ISSN

0316-0041 (imprimé)

1492-1367 (numérique)

Découvrir la revue

Citer cet article

Gendron, A., Admo, N., Plourde, C. \& Thibault, I. (2020). Enjeux du travail policier en milieux autochtones au Québec : la méthode d'analyse en groupe au service de la recherche de solutions. Criminologie, 53(2), 335-358.

https://doi.org/10.7202/1074198ar

\section{Résumé de l'article}

Bien que quelques auteurs se soient penchés sur la police autochtone, très peu, voire aucun, ne s’y sont intéressés dans une visée compréhensive et qualitative à partir du point de vue des policiers. Adoptant une perspective participative, cette étude vise à expérimenter la méthode d'analyse en groupe auprès de sept policiers issus de corps de police autochtones québécois pour documenter les principaux enjeux du travail en milieux autochtones et formuler des pistes de solutions adaptées aux réalités des communautés. Les résultats mettent notamment en évidence des enjeux liés à la non-reconnaissance de leur rôle au sein de la communauté autochtone et même policière, au manque de soutien organisationnel, à la proximité citoyenne et politique, à l'isolement social et professionnel, ainsi qu'à l'accessibilité lacunaire aux services partenaires pour soutenir leurs interventions. Ces défis se juxtaposent aux importants problèmes sociaux auxquels ces policiers font face quotidiennement. Cette démarche donne lieu à des pistes de solutions reposant sur la variété de représentations, lesquelles ont également permis l'élaboration d'un projet de recherche visant à approfondir la compréhension de la perception des policiers intervenant auprès des Premières Nations, Métis ou Inuits. 


\title{
Enjeux du travail policier en milieux autochtones au Québec
}

\section{La méthode d'analyse en groupe au service de la recherche de solutions}

\author{
Annie Gendron ${ }^{1,2}$ \\ Chercheure, École nationale de police du Québec \\ Chercheure régulière, Centre international de criminologie comparée \\ Professeure associée, Département de psychoéducation, Université du Québec à Trois-Rivières \\ annie.gendron@enpq.qc.ca \\ Nina Admo \\ Professeure chercheure, Collège de Maisonneuve \\ Chercheure régulière, Centre international de criminologie comparée \\ nadmo@cmaisonneuve.qc.ca \\ Chantal Plourde \\ Professeure chercheure, Département de psychoéducation, Université du Québec à Trois-Rivières \\ Chercheure régulière, Centre international de criminologie comparée \\ chantal.plourde@uqtr.ca

\section{Isabelle Thibault} \\ Candidate au doctorat en psychologie, Université du Québec à Trois-Rivières \\ Étudiante, Centre international de criminologie comparée \\ isabelle.thibault2@uqtr.ca
}

RÉSUMÉ - Bien que quelques auteurs se soient penchés sur la police autochtone, très peu, voire aucun, ne s'y sont intéressés dans une visée compréhensive et qualitative à partir du point de vue des policiers. Adoptant une perspective participative, cette étude vise à expérimenter la méthode d'analyse en groupe auprès de sept policiers issus de corps de police autochtones québécois pour documenter les principaux enjeux du travail en milieux autochtones et formuler des pistes de solutions adaptées aux réalités des communautés. Les résultats mettent notamment en évidence des enjeux liés à la nonreconnaissance de leur rôle au sein de la communauté autochtone et même policière,

1. École nationale de police du Québec, 350, rue Marguerite-D’Youville, Nicolet (Québec), Canada, J3T 1X4.

2. Ces travaux ont été financés par le Conseil de recherches en sciences humaines (CRSH).

Criminologie, vol. 53, $\mathrm{n}^{\circ} 2(2020)$ 
au manque de soutien organisationnel, à la proximité citoyenne et politique, à l'isolement social et professionnel, ainsi qu'à l'accessibilité lacunaire aux services partenaires pour soutenir leurs interventions. Ces défis se juxtaposent aux importants problèmes sociaux auxquels ces policiers font face quotidiennement. Cette démarche donne lieu à des pistes de solutions reposant sur la variété de représentations, lesquelles ont également permis l'élaboration d'un projet de recherche visant à approfondir la compréhension de la perception des policiers intervenant auprès des Premières Nations, Métis ou Inuits.

MotS CLÉS • Méthode d'analyse en groupe, intervention policière, autochtones, enjeux.

\section{Contexte théorique}

Traditionnellement, le maintien de la paix, de l'ordre et de la sécurité publique dans les communautés autochtones (Premières Nations, Inuits et Métis) ${ }^{3}$ était pris en charge par les dirigeants communautaires: les Aînés, les guerriers, les familles ou d'autres membres de la communauté (Jones, Mills, Ruddell et Quinn, 2016). Ces pratiques se sont peu à peu estompées en 1870 avec la création de la police étatique. À partir de 1960, à la suite de nombreuses revendications provenant des conseils de bande, une partie des services policiers est dorénavant assurée par des agents de bande, puis par des constables spéciaux désignés par la Gendarmerie royale du Canada (Aubert et Jaccoud, 2009). En 1978, un premier service de police autochtone est légalement constitué, le Service de la Police amérindienne, mais ses pouvoirs sont très limités. Ensuite, le gouvernement fédéral adopte en 1991 la Politique sur la police des Premières Nations (PPPN), qui privilégie l'autodétermination des communautés autochtones en matière de police sur le terrain (Aubert et Jaccoud, 2009). Un des principaux objectifs visés est de permettre aux Autochtones une participation à l'administration de la justice culturellement adaptée. La PPPN se veut également une façon d'uniformiser les forces policières autochtones en matière de formation, d'assermentation des officiers et des pouvoirs accordés (Kiedrowsky, Jones et Ruddell, 2017). Contrairement aux autres corps de police québécois, qui relèvent entièrement du ministère de la Sécurité publique du Québec, la majorité des corps de police autochtones, en conformité avec

3. La Constitution canadienne stipule que les «peuples autochtones du Canada» s'entend notamment des Indiens (Premières Nations), des Inuits et des Métis du Canada (article $35(2)$ ). Nous reconnaissons que chaque communauté a sa propre histoire, ses propres langues, pratiques culturelles et croyances. Nous utilisons le terme «Autochtones» ou «peuples/communautés autochtones» pour désigner les Premières Nations, les Inuits et les Métis. 
l'article 90 de la Loi sur la police du Québec, est autogérée par l'entremise des conseils de bande et dépendent financièrement des gouvernements fédéral et provincial (Jaccoud et Spielvogel, 2018; Kiedrowski, Petrunik et Ruddell, 2016; Sécurité publique Canada, 2016). À l'exception des corps policiers des nations cries, inuites et du village de Naskapis, qui sont conventionnés ${ }^{4}$, les autres corps policiers autochtones négocient sur une base récurrente des ententes individuelles. Au Québec, 51 des 55 communautés autochtones visées par des ententes sur la prestation de services policiers rejoignent 99,5\% de la population autochtone (Ministère de la Sécurité publique, 2017). Les autres communautés dépendent de la Sûreté du Québec, qui a aussi le mandat de soutenir tous les autres corps policiers municipaux québécois. Ainsi, malgré la volonté de la PPPN d'uniformiser les services de police desservant les collectivités autochtones, des inégalités persistent, affectant leur capacité d'autodétermination en matière policière (Jaccoud et Spielvogel, 2018). Plusieurs communautés autochtones sont d'ailleurs insatisfaites du caractère partiel de l'autonomie retrouvée (Canadian Association of Police Governance, 2016).

L'insatisfaction des communautés autochtones est également liée au système de justice pénale comme mode de traitement des situations problématiques qui entre en conflit avec les traditions et coutumes davantage orales que procédurales de ces communautés (Aubert et Jaccoud, 2009; Lithopoulos et Ruddell, 2013). Aujourd'hui, les modèles de règlements des différends basés sur les traditions autochtones demeurent marginaux (Chartrand et Horn, 2016), et ce, malgré la mise en place du Programme de justice autochtone par le gouvernement canadien. Ensuite, l'insatisfaction des Autochtones est aussi liée à une perception d'inefficacité de la police à contrôler adéquatement la criminalité dans leur communauté (Cao, 2014; Jones, Ruddell et Summerfield, 2019). Plusieurs Autochtones entretiennent l'idée que les désordres sociaux vécus dans les communautés «sont l'indice résiduel de l'État malsain et de déséquilibres que la colonisation a produits» (Jaccoud, 1999, p. 87), notamment par les pensionnats autochtones (Jones et al., 2019; Ruddell et Lithopoulos, 2013) et l'appropriation de leurs terres par l'État. Encore aujourd'hui, les traumatismes liés aux processus d'assimilation et d'acculturation (Jones et al., 2019;

4. Les corps policiers conventionnés ne sont pas régis par la Loi sur les Indiens, mais soumis au même régime fiscal que l'ensemble de la population québécoise et ils ne bénéficient d'aucune exemption particulière. 
McDonough, 2013; Monchalin, 2016) sont bien présents dans la mémoire collective des Autochtones (Aguiar et Halseth, 2015). Ils s'actualisent, entre autres, par le peu de légitimité accordée aux actions des représentants de l'État et par le maintien de relations tendues avec ces derniers (Salée, 2005). Les policiers sont toujours perçus comme étant des représentants du pouvoir étatique (LeBeuf, 2011) pour contrôler les populations autochtones (Tulloch, 2017), qui se disent victimes de racisme et de discrimination systémique (Brassard, Spielvogel et Montigny, 2016; Monchalin, 2016; Posca, 2018). Plusieurs chercheurs notent qu'une large part des contrôles et des arrestations est en effet réalisée de manière arbitraire, notamment en milieux urbains (Aubert et Jaccoud, 2009; Cao, 2014; Jaccoud et Spielvogel, 2018). La surreprésentation des Autochtones dans les institutions carcérales (Brassard et al., 2016; Chartrand, 2019; Roberts et Reid, 2017) est une autre manifestation de cette discrimination systémique et du profilage racial dont ils sont victimes (Fitzgerald et Carrington, 2008; Jaccoud et Spielvogel, 2018, La Prairie, 2002). Un rapport de force inégal réside déjà dans la relation entre citoyens et policiers, et ce, par les pouvoirs et devoirs délégués au policier. Mais pour plusieurs citoyens autochtones, les déséquilibres dans cette relation sont aussi occasionnés par les victimisations passées et par la discrimination systémique.

De plus, les traumatismes majeurs occasionnés par les politiques visant l'assimilation des Premières Nations, Inuits et Métis tout au long des $\mathrm{XIX}^{\mathrm{e}}$ et $\mathrm{xx}^{\mathrm{e}}$ siècles s'observent aussi par plusieurs enjeux sociaux persistants au sein des communautés: la précarité économique et alimentaire, la pauvreté, les logements insalubres, la détresse psychosociale, l'itinérance et les dépendances aux substances (Bellot et Sylvestre, 2016; Commission de la santé et des services sociaux des Premières Nations du Québec et du Labrador, 2018; Jaccoud et Spielvogel, 2018). À cela s'ajoutent la prévalence importante des comportements délictuels et l'expression d'une violence excessive (Brzozowski, Taylor-Butts et Johnson, 2006; Chrismas, 2012). Les policiers font régulièrement face à des situations complexes sans pour autant toujours posséder le mandat ou les compétences nécessaires pour les résoudre (Griffiths et Clark, 2017; Salée, 2005). De nombreuses embûches attendent les policiers appelés à intervenir dans ces communautés, auxquelles s'ajoutent celles liées à l'apparence d'impartialité, notamment lorsque le policier doit intervenir auprès de sa propre famille (Jones et al., 2016). D'ailleurs, bon nombre de policiers ont l'impression que les problèmes de rétention 
des effectifs policiers en milieux autochtones sont propres à l'environnement professionnel de ces communautés (Lithopoulos et Ruddell, 2011 ; Ruddell et Jones, 2018). L'insuffisance du financement des corps policiers autochtones, en plus de rendre l'accès à la formation difficile, contribue largement à la précarité des conditions de travail (Aubert et Jaccoud, 2012). Tous ces enjeux sont source de tensions entre les citoyens autochtones et les policiers (Aubert et Jaccoud, 2009) et génèrent bien souvent des situations de «crise». Les allégations d'agressions sexuelles envers des femmes autochtones par des policiers ont également contribué à exacerber les difficultés relationnelles entre les policiers et les Autochtones (Enquête nationale sur les femmes et les filles autochtones disparues et assassinées, 2017). L'ensemble de ces défis appuie la nécessité d'améliorer les pratiques en matière d'intervention policière auprès d'Autochtones (Commission d'enquête sur les relations entre les Autochtones et certains services publics: écoute, réconciliation et progrès, 2019).

Malgré ces constats, le travail policier en communautés autochtones au Canada demeure l'un des aspects les moins étudiés (Jaccoud et Spielvogel, 2018; Jones et al., 2019; Ruddell et Lithopoulos, 2013). Les travaux menés jusqu'à maintenant se sont surtout penchés sur le vécu des Autochtones en lien avec les interventions policières, et non avec des policiers eux-mêmes (Jones et al., 2019). Bien que quelques chercheurs se soient concentrés sur cette question (Jaccoud et Splielvogel, 2018; Jones et al., 2016; Lithopoulos et Ruddell, 2018), très peu, voire aucun, ne s'y sont intéressés sous l'angle du vécu des policiers, de leurs représentations du métier, de la formation reçue et de leurs besoins en contexte d'intervention. Cette étude exploratoire vise donc à mieux comprendre la réalité des policiers œuvrant dans les communautés autochtones, et ce, par le recours à une méthode impliquant les acteurs concernés à toutes les étapes de la recherche. Plus précisément, la méthode d'analyse en groupe (MAG) décrite plus loin a été utilisée pour documenter les particularités du travail policier dans ces communautés à partir de témoignages de policiers autochtones et allochtones, et pour formuler des pistes de solution adaptées à leurs réalités.

\section{Cadre méthodologique}

La compréhension de la réalité des policiers œuvrant en communautés autochtones justifie le recours à une approche qualitative pour mettre 
en relief le sens que les personnes donnent à leurs expériences (Mucchielli et Paillé, 2003). Cette recherche s'inscrit dans le cadre de la théorisation enracinée (grounded theory) de Glaser et Strauss (1967) dans laquelle les participants sont considérés comme étant des «sujets connaissants» et non des «objets de connaissance». Corbin (2012) explique que la finalité de la théorisation enracinée est de saisir la variabilité et la complexité des phénomènes humains dans le but de générer une théorie à partir de données empiriques. Cela implique une démarche de validation qui prévoit des mouvements itératifs et circulaires entre les moments de collecte et d'analyse des données, lesquels engagent la contribution des répondants à toutes les étapes du processus (Guillemette et Lapointe, 2012). Dans le cadre de cette étude, la MAG a été utilisée afin d'explorer les enjeux rencontrés par des policiers œuvrant au sein d'un corps de police autochtone. Cette approche basée sur le savoir expérientiel mène à une théorisation à partir d'expériences vécues. La MAG repose donc sur sa capacité à produire des données nouvelles dans une logique inductive et participative, assurant la fidélité et la validité des théories émergentes, mais aussi des solutions proposées par les acteurs impliqués.

La MAG, telle qu'elle est définie par Van Campenhoudt, Chaumont et Franssen (2005), se rapproche de l'entretien de groupe, à l'exception du déroulement qui est très structuré et des retombées qui ont des visées autant théoriques que pratiques (Konaté et al., 2019). Elle est composée d'étapes permettant de faire ressortir les éléments centraux liés à l'objet d'étude par un processus d'analyse en groupe à partir de récits d'évènements. Cette façon de procéder permet à tous les participants d'exprimer leur point de vue et de contribuer à l'interprétation des problèmes et enjeux exprimés dans les récits. Dans ce processus, les chercheurs tiennent les rôles d'animateur ou de preneur de notes, laissant les participants au premier plan, ce qui diffère du groupe de discussion classique.

Notre équipe a structuré la MAG en quatre étapes réparties sur deux jours. Ainsi, à l'étape 1 , chaque participant expose brièvement à tour de rôle le récit d'un évènement vécu témoignant de ses préoccupations liées à l'objet d'étude. Ces récits devaient être réfléchis à l'avance, comme spécifié dans les consignes acheminées préalablement. Deux récits, les plus significatifs aux yeux des participants, sont retenus par vote au début de la MAG pour faire l'objet du processus de théorisation. Lors de l'étape 2, chaque participant communique son interprétation 
du premier récit retenu qui a été relaté en détail par le narrateur (participant ayant vécu l'évènement). Les convergences et divergences notées dans les échanges sont ensuite mises en évidence à l'étape 3 par les chercheurs sous forme d'un schéma synthèse (modélisation). La modélisation est une étape de validation cruciale reposant sur les bases empiriques ayant émergé de l'interprétation du problème abordé par les participants. Il ne s'agit pas d'obtenir un consensus, mais d'amener les participants à revoir la problématique en formulant de nouvelles perspectives à partir du travail collectif effectué, et de saisir l'ensemble des liens entre les points de vue. À la suite de la modélisation et avant d'entamer l'étape 4, les chercheurs présentent un éclairage théorique, issu de la littérature, sur les thématiques ayant émergé de l'analyse collective. Dans l'étape 4, les participants tentent de concevoir des pistes de solutions en considérant les connaissances théoriques présentées. Ensuite, un deuxième récit est choisi et soumis au processus d'analyse en quatre étapes. La démarche se termine par une synthèse où les participants valident la compréhension des chercheurs quant à l'objet d'étude selon leurs perspectives. La richesse de cette démarche repose sur le fait que les pistes de réponses proposées sont non seulement tirées d'une analyse collective des personnes expérimentant directement le problème, mais aussi de leur adéquation avec les réalités sur le terrain. Comme le préconise la théorisation enracinée (Corbin, 2012), le processus de recherche s'inscrit dans une démarche de validation continue du début à la fin.

\section{Participants et procédures}

Pour expérimenter la MAG, l'invitation a été transmise à 14 corps policiers autochtones du Québec dont la principale langue utilisée en contexte de travail est le français ${ }^{5}$. De ce nombre, sept corps policiers autochtones non conventionnés ont accepté de dégager une ressource volontaire pour prendre part aux travaux. Le groupe se compose donc de sept policiers ayant une expérience variant de 5 à 30 années de service. Parmi eux, cinq policiers sont d'origine autochtone et deux sont allochtones. On trouve six hommes et une femme. Afin d'amenuiser

5. Puisqu'il s'agissait d'une étude pilote visant à tester la MAG en contexte policier, la langue française a été privilégiée. De futurs travaux sont prévus avec les autres corps policiers intervenants auprès de clientèles autochtones, incluant les corps policiers autochtones conventionnés. 
toute possibilité d'identification des participants, le masculin est utilisé pour la présentation des extraits. Comme il avait été convenu lors du recrutement, aucune compensation financière ne leur a été offerte; seuls leurs frais de séjour et de déplacement ont été pris en charge lorsque non assumés par le corps de police. La rencontre de deux jours s'est tenue dans une salle de réunion de l'École nationale de police du Québec en raison de sa position géographique centralisée.

Une semaine avant la MAG, les consignes ont été acheminées à tous les participants ayant signifié leur intention de prendre part aux travaux. Il était spécifié qu'ils devaient préparer un récit relatant une situation vécue à titre de policier exposant un problème relié à l'objet d'étude. Au moment d'entreprendre la MAG, l'étude et ses objectifs ont été réexpliqués aux participants à qui un formulaire de consentement a été distribué. Les échanges ont été enregistrés puis retranscrits de façon anonymisée ${ }^{6}$ afin de cibler des extraits parlants pour mieux rapporter les propos des participants.

\section{Résultats}

L'analyse collective réalisée a permis de faire émerger les principaux enjeux rencontrés par ces policiers œuvrant au sein de communautés autochtones. Le premier récit retenu fait référence à une situation où l'influence politique aura eu un impact important sur la carrière d'un participant à la suite de la mort d'un citoyen lors d'une intervention policière. Pour sa part, le second expose un important décalage entre la réponse des services de soutien en matière de protection de l'enfance et les besoins policiers en situation d'urgence. Les propos des participants dans l'analyse de ces deux récits s'articulent autour de six enjeux: le manque de reconnaissance, le manque de soutien organisationnel, la proximité politique, l'isolement professionnel et social, la multitude des problèmes sociaux récurrents dans les communautés et le manque d'accès aux services partenaires en sécurité publique.

6. Le protocole a été approuvé par le comité d'éthique et de la recherche de l'Université du Québec à Trois-Rivières (CER-17-239-07.14) ainsi que par le comité d'éthique du Collège de Maisonneuve. 


\section{Le manque de reconnaissance}

Les participants ayant pris part à la MAG mettent en lumière une particularité de la réalité du travail policier en communautés autochtones qui serait, selon eux, au cœur des autres enjeux exposés: le manque de reconnaissance. Les participants estiment que les corps de police autochtones sont victimes d'un manque de reconnaissance chronique et parlent même de discrimination systémique de la part du gouvernement et parfois même de la part de collègues policiers au sein des services allochtones. Malgré toutes les transformations apportées à la police autochtone au fil des années, et la mise en place de la PPPN, ces corps policiers demeurent une classe à part comportant de multiples inégalités. Au Québec, des niveaux de service variant de 1 à 6 sont attribués aux corps policiers allochtones selon la taille de la population qu'ils desservent ou leur localisation géographique (Ministère de la Sécurité publique, 2020). Ces niveaux impliquent des responsabilités opérationnelles et un financement adapté. Puisque les corps policiers autochtones non conventionnés sont tributaires des ententes tripartites, ils ne sont pas intégrés à ce système de niveaux, illustrant par le fait même le manque de reconnaissance avancé par les participants de la MAG:

Notre statut autochtone, dans les grandes machines de 15000 policiers au Québec, on est traités comme [petit rire], on n'est pas de niveau 1 [en référence aux niveaux de services]. Près de 40 ans de police autochtone et on est toujours le niveau 0 des organisations. On n'est pas reconnus comme polices, on est toujours des $3 / 4$ ou des $7 / 8$. (Participant 2, 18 ans de service)

Selon les participants, la non-reconnaissance s'exprime aussi par l'entremise de différents défis en matière de ressources humaines: moins bons salaires, formation continue limitée, équipements désuets, etc. En effet, le sous-financement chronique de ces services policiers les empêche d'offrir des salaires concurrentiels, ce qui entraîne aussi des difficultés importantes en termes de recrutement et de rétention du personnel (Aubert et Jaccoud, 2012; Kiedrowsky et al., 2017). Un rapport ministériel déposé en 1973 avait déjà souligné que le financement des services policiers autochtones était insuffisant et inéquitable (Kiedrowsky et al., 2017). Une étude plus récente rapportant des données collectées en 2009 auprès de directeurs de corps policiers autochtones avait aussi mis en lumière les conditions de travail précaires dans lesquelles ils doivent exercer leur mandat (Aubert et Jaccoud, 2012). Force est d'admettre que dix ans plus tard, ce constat est toujours d'actualité. 
Lorsqu'ils se comparent aux policiers ailleurs au Québec, les participants à la MAG perçoivent que leurs conditions de travail sont nettement inférieures:

Tu sais, on n'est pas reconnus au même titre que les autres policiers ailleurs au Québec dans les grandes villes. Eux autres ils sont à $50 \$$ de l'heure après 6 ans, mais moi j'ai des amis que ça fait 20-25 ans qu'ils sont dans la police, et ils sont à $20 \$$. Tu sais quand j'ai commencé dans l'autopatrouille, j'avais $15 \$$ de l'heure. Ça démontre un peu qu'on est une classe à part. (Participant 3,8 ans de service)

Bien que la PPPN poursuivait l'objectif d'uniformiser la formation, le cercle vicieux qu'entraîne le sous-financement des corps policiers autochtones limite nécessairement les possibilités de recrutement, mais aussi de formation (Kiedrowsky et al., 2017). Il ne s'agit pas ici de remettre en question la qualité des formations offertes ou de se demander si elles sont culturellement adaptées, mais plutôt de considérer la difficulté pour des policiers autochtones d'y accéder (Jaccoud et Spielvogel, 2018) comme en témoigne l'extrait suivant:

Nos organisations policières, ce n'est pas de la mauvaise volonté de dire non, on veut former nos policiers, mais un moment donné... L'argent... Le mot se passe auprès des autres organisations policières, et qu'est-ce que ça lance comme message? "Ah, sont pas formés eux autres.» Euh... un instant, là! Ce n'est pas de la mauvaise volonté, au contraire, on voudrait bien. (Participant 6, 9 ans de service)

Selon les propos des participants, les difficultés d'accès à la formation entraînent à leur tour le manque de reconnaissance qu'ils perçoivent, cette fois, de la part de leurs collègues policiers œuvrant au sein de corps de police allochtones:

À cause qu'on est Autochtone, j'ai fait une attestation d'études collégiales [ 1 an d'études techniques], je n'ai pas le diplôme d'études collégiales [3 ans d'études techniques], mais j'ai fait le même 15 semaines de l'École de police comme les autres. C'est juste qu'il a été adapté à nous autres. Pourquoi les autres policiers ne sont pas capables de le reconnaitre? Comme si je n'étais pas un policier, comme si j'étais un... un agent de sécurité. (Participant 5, 6 ans de service)

L'ensemble de ces affirmations amène les participants à parler d'une forme de discrimination systémique au sein même de la communauté policière qui prend racine dans une non-reconnaissance initiale des services de police autochtones. Les multiples obstacles organisationnels 
qui en découlent contribuent à creuser le fossé avec leurs homologues allochtones et semblent parfois les placer dans une position d'infériorité, perçue ou réelle, par rapport à ces derniers.

\section{Le manque de soutien organisationnel et la proximité politique}

Les participants à la MAG expliquent également qu'ils doivent évoluer dans un environnement où le soutien organisationnel est lacunaire, et parfois même absent. Cette perception est notamment expliquée par la position souvent délicate dans laquelle se trouve le gestionnaire qui doit remplir son mandat en supportant les différentes pressions politiques locales. Les participants perçoivent une proximité politique qui crée une pression importante, pour ne pas dire de l'ingérence, au cœur même de leurs interventions:

Le conseil de bande fait tellement des pressions, des manifestations, ils ramènent tout le temps ça aux pensionnats: vous nous enlevez nos enfants. Fait qu'un moment donné pour calmer le jeu, ils disent c'est beau, on va essayer de trouver une famille ici. On le sait que ce n'est pas la meilleure, mais on va calmer la tempête. Est-ce que c'est vraiment ce qui est bon pour l'enfant? Je ne pense pas. (Participant 3, 8 ans de service)

Kiedrowsky et al. (2017) expliquent que l'ingérence politique entraîne l'instabilité des opérations policières, et que cette instabilité est renforcée par le manque de soutien des dirigeants. Selon nos participants, la proximité politique est un facteur important qui contribue à fragiliser le soutien organisationnel, car cette pression s'exerce sur toutes les strates de l'organisation:

Après l'évènement tragique, il n'a pas eu le soutien de sa direction, et même pas de ses confrères de travail. Il a été pratiquement laissé à lui-même. Ça, ça vient me rejoindre, de ne pas avoir ce soutien-là, par son conseil de bande aussi. (Participant 7, 30 ans de service)

Dans le fond, c'est de penser à ta carrière quand tu le sais très bien que tu ne seras pas «backé» par ta haute direction ni au niveau du conseil s'il arrive quelque chose. (Participant 4, 9 ans de service)

Les participants proposent l'image de l'épée de Damoclès pour illustrer comment ils se sentent lorsqu'ils enfilent leur uniforme de policier. Pour eux, une intervention policière qui tourne mal peut représenter une fin de carrière. Ils expliquent cette perception par les 
pressions politiques locales qu'ils risquent de subir après l'évènement, l'effritement des relations avec les membres de la communauté, des perspectives limitées en matière d'emploi ou de mutation d'un corps de police vers un autre. Il est effectivement connu que les difficultés d'avancement et de mobilité sont préoccupantes au sein des corps policiers autochtones (Ministère des Affaires indiennes et du Nord canadien, 1990). Les participants soutiennent que cet enjeu leur est propre alors que les services de police allochtones bénéficieraient de plus de possibilités d'emplois:

C'est que si on perd notre travail parce qu'il arrive un évènement, je ne serai pas replacé ailleurs comme dans la Sûreté du Québec par exemple. Ils vont dire: «Trouve-toi un job ailleurs.» Dans la police, quand on est célibataire pas d'enfant, des fois ça ne nous dérange pas d'aller ailleurs. Quand tu as quatre enfants et une femme et que notre famille est aux alentours, bien tu dois faire un choix. (Participant 6, 9 ans de service)

Le manque de soutien organisationnel est donc perçu localement, mais également au sein même du système policier, entre autres, en raison de la position géographique souvent éloignée des communautés. Une mutation implique fréquemment un déracinement de la famille entière, ce qui n'est pas forcément le cas pour les policiers des corps de police allochtones, notamment dans les grands centres urbains (Ruddell, Lithopoulos et Jones, 2014).

\section{L'isolement professionnel et social}

En plus du manque de reconnaissance et de soutien organisationnel, les participants de la MAG parlent d'un fort sentiment d'isolement professionnel et social. Tout d'abord, cet isolement professionnel est expliqué en partie par le contexte géographique de certaines communautés éloignées. Les participants se perçoivent en situation de vulnérabilité importante advenant un évènement majeur, en comparaison de leurs collègues en milieux allochtones:

Si on a besoin d'aide, il n'y en a pas de back-up! Vous êtes juste deux! Deux contre le village. S'il arrive de quoi de gros... le temps que le monde arrive, et que les avions arrivent... Est-ce que l'avion va décoller? S'il fait tempête, t'es tout seul avec ton collègue! (Participant 5, 5 ans de service)

Les participants expliquent aussi le sentiment d'isolement sous l'angle social, lequel serait une des conséquences de la proximité 
citoyenne. Leurs discours témoignent d'une certaine forme de «personnalisation des interventions policières», soit d'une difficulté perçue chez le citoyen à dissocier l'individu et son rôle de policier. Cette personnalisation semble plus lourde pour ces participants œuvrant en communautés autochtones et contribue grandement à leur isolement social:

C'est-à-dire que si vous travaillez comme policier dans la ville $\mathrm{A}$, que vous exécutez un mandat, il va passer. Nous, si on exécute un mandat, c'est envers un membre de la communauté. Quand t'appliques un mandat sur une personne qui est un fils ou... une personne bien connue politiquement, la répercussion n'est pas la même qu'un policier qui travaille dans la ville $A$, vous êtes celui qui a appliqué le mandat. Je m'aperçois que mon cercle d'amis après 9 ans, il a réduit énormément. (Participant 6, 9 ans de service)

On me dit: «C'est toi qui es connu ici pour faire des signalements, personne t'aime à cause de ça.» Moi bien, je fais juste mon job. Même mon frère des fois il me le dit: «Personne t'aime ici sur la communauté.» (Participant 1, 8 ans de service)

Les tensions perçues entre les policiers et les citoyens autochtones constituent une autre dimension déterminante de leur réalité professionnelle. Encore aujourd'hui, les traumatismes liés aux processus d'assimilation et d'acculturation décrits dans plusieurs études (McDonough, 2013; Monchalin, 2016; Jones et al., 2019) s'ajoutent aux nombreuses pressions propres au contexte de travail des policiers en communautés autochtones. Les représentations négatives à l'égard de la police chez plusieurs Autochtones contribuent à l'isolement social de ces professionnels. Finkler (1980) avait relevé il y a 40 ans que les policiers inuits étaient ostracisés par les gens de la communauté. Les policiers autochtones sont aussi victimes de rejet et de discrimination par les gens de la communauté (Griffiths et Yerbury, 1984). Selon les propos des participants de la MAG, le problème demeure entier et entraîne d'importantes répercussions psychologiques et sociales pouvant même mener à l'abandon de la carrière policière.

Toutefois, les participants sont tous d'accord pour dire que la proximité citoyenne peut parfois avoir des aspects positifs, notamment lorsqu'ils doivent pallier le manque de ressources policières lors d'interventions délicates. Cette proximité peut, dans certaines circonstances, améliorer l'efficacité des interventions (Jaccoud et Spielvogel, 2018). L'extrait suivant témoigne d'une circonstance où la proximité a permis d'éviter le pire: 
On s'est déjà fait entourer par une quarantaine de personnes lors d'une arrestation. Il y en a qui nous lançaient des bouteilles de bière. Puis tu regardes dans le tas, c'est qui dans le gang qui va t'aider, et tu lui cries: "Aide-nous.» C'est le fun parce que tu reconnais quelqu'un et que tu as déjà été comme cool avec lui, t'as été moins sévère. Tu le regardes et puis il dit au monde: «O.K.! c'est beau, reculez, arrêtez.» Ça aide beaucoup quand tu connais du monde. (Participant 1, 8 ans de service).

\section{La multitude des problèmes sociaux}

À ces enjeux systémiques s'ajoute un contexte de travail très souvent complexe. L'ampleur des problèmes sociaux dans les communautés autochtones, la plupart du temps récurrents, ajoute aux difficultés perçues par ces participants. À travers ces interventions délicates, les impacts de la proximité citoyenne prennent tout leur sens:

J'ai eu à intervenir à plusieurs reprises chez ma tante pour des violences conjugales et de la consommation. Puis je suis le parrain de la petite fille, la dernière. J'ai fait beaucoup de signalements. Après 5 ou 6 interventions, je suis allé à la cour. Je me suis débattu pour que la garde soit enlevée. (Participant 1, 8 ans de service)

Les résultats de l'étude de Kiedrowsky et al. (2017) menée auprès de policiers autochtones rapportent que ces derniers sont appelés à gérer des problèmes sociaux qu'ils considèrent comme insolvables, dont la violence intrafamiliale, la consommation d'alcool et de drogues, et la santé mentale. De surcroît, l'accès lacunaire à la formation continue de creuser l'iniquité entre leurs conditions de travail et celles des autres policiers québécois. Mais ces problèmes d'accès contribuent aussi à limiter la consolidation des acquis et la bonification des interventions policières dans des milieux de vie aux prises avec des problèmes sociaux sérieux et intergénérationnels.

\section{Le manque d'accès aux services partenaires}

En pareil contexte, l'accès aux services partenaires en sécurité publique est essentiel, tant pour soutenir l'intervention policière que pour offrir aux citoyens les services nécessaires adaptés à leurs besoins. Or, les participants rencontrés affirment tous que l'accessibilité déficiente aux services de soutien représente un poids important qui ajoute une pression indue sur leur contexte de travail en communautés autochtones. 
Lors de la MAG, ils ont surtout abordé les difficultés rencontrées lorsqu'ils doivent faire appel à la Direction de la protection de la jeunesse, en raison du deuxième récit retenu pour l'analyse impliquant ce partenaire. Comme en témoigne cet extrait, l'accès aux ressources psychosociales en matière de protection de l'enfance est difficile et semble parfois même associé, dans l'esprit des participants, au manque de reconnaissance précédemment décrit:

Les intervenants sociaux dans les communautés, quand tu fais des signalements, ils ne les retiennent jamais. Ils ne se déplacent pas pour valider les informations. Cette journée-là, j'ai appelé la DPJ. L'intervenante a répondu qu'elle va venir durant la semaine. Fait que j'ai dit: «Non! tu n'attends pas toute la semaine, tu t'en viens tout de suite.» Elle a répondu: «Non! On a des protocoles à respecter.» «Je m'en fous de tes protocoles, tu t'en viens voir la maison, c'est... absurde. La maman est trop intoxiquée, on n'est pas capable de la réveiller. Il y a beaucoup trop d'objets au sol qui peuvent blesser un enfant. Il n'y a pas de nourriture, il n'y a absolument rien, il y a un bébé qui pleure dans ses couches souillées, tu te déplaces tout de suite.» (Participant 1, 8 ans de service)

Les participants à la MAG en viennent même à se demander si les difficultés vécues avec les services partenaires, surtout la DPJ dans le contexte, ne seraient pas aussi le résultat d'une discrimination à l'égard des Autochtones:

Ils ne veulent jamais se déplacer. On dirait que la DPJ ne veut pas. Je ne sais pas, c'est-tu à cause qu'on est Autochtones... ? C'est quoi les causes réelles... ? On n'a pas le temps... ou peut-être qu'ils doivent se dire: «Ah! ça fait cinq fois qu'on y va, là, pis c'est tout le temps la même histoire.» (Participant 5, 5 ans de service)

Spontanément, les participants apportent néanmoins des pistes pour expliquer cette difficulté particulière qu'ils doivent fréquemment affronter. Ils soulèvent un manque possible de ressources du côté de la DPJ ou encore un manque de compréhension des réalités de chacun:

Je pense que peut-être, c'est une question de priorités. On classe tous un peu nos dossiers par ordre prioritaire, peut-être qu'ils ne sont pas assez... tu pars du pire dossier, et tu te rends jusqu'à où que tu peux fournir. (Participant 3, 8 ans de service)

Moi de mon côté, je pense que c'est un très grand manque de communication et de connaissance du problème. D'un côté comme de l'autre, on s'imagine un peu c'est quoi le travail de la DPJ, c'est quoi leur protocole, tout ça, mais sans nécessairement savoir c'est quoi leur façon de faire. Mais 
c'est la même chose de leur côté aussi à propos de notre travail. (Participant 6, 9 ans de service)

Il reste que lorsque l'on considère le témoignage en lien avec la mère inconsciente intoxiquée et le bébé en pleurs, force est de constater que les services sociaux peinent en effet à subvenir aux besoins urgents de ces collectivités et à soutenir les policiers dans leurs interventions.

\section{Des dimensions centrales malgré tout: fierté et attachement}

Au cour de cette vue d'ensemble et malgré les nombreux défis et embûches rencontrés, les participants mettent de l'avant l'aspect vocationnel de leur profession et des sentiments soutenus d'attachement et de fierté:

Je suis bien fier de faire ça, d'habiter là. Il y a des côtés négatifs, des choses

à améliorer, mais il y a aussi de belles choses qui sont faites. (Participant

6, 9 ans de service)

Cette dimension vocationnelle, qui semble inébranlable pour les participants de la MAG, peut agir selon nous de tremplin dans la recherche de pistes de solutions aux nombreux enjeux qu'ils doivent affronter. Il reste que cette vocation est ébranlée par l'impression d'être isolés, peu reconnus et jugés de tous. Quelconque piste de solution devra également intégrer ces dimensions propres au travail des policiers dans certaines communautés autochtones.

\section{Pistes de solutions}

Dans le processus d'analyse, la mise en évidence des enjeux soulevés par les participants a donné lieu à une meilleure compréhension des réalités du travail policier en communautés autochtones. Sur cette base, les participants à la MAG ont pu formuler des suggestions à propos de la structure même des corps de police autochtones ainsi que leur financement, les aspects opérationnels et la formation. Bien que ces suggestions n'aient pas été confrontées au test de la faisabilité, elles répondent à des préoccupations contemporaines des acteurs du terrain. Comme le suggèrent Lithopoulos et Ruddell (2011) ainsi que Ruddell et Jones (2018), les difficultés de ces corps de police sont propres à l'environnement professionnel qui diffère grandement de ceux de leurs collègues en milieux allochtones. La recherche de 
solutions doit donc s'inscrire à l'intérieur même de cet environnement atypique.

Concernant les suggestions structurelles, les participants ont rapporté devoir travailler malgré d'importantes lacunes financières qui se traduisent par une inégalité des conditions de travail et un manque de ressources humaines et matérielles. Comme déjà mis en lumière par Clairmont et Murphy (2000), le sous-financement chronique place les corps policiers autochtones en situation d'échec. Ainsi, les participants suggèrent de revoir le principe de l'entente tripartite de financement des corps de police autochtones. Selon leurs points de vue, ceci permettrait d'assurer la pérennité des services et d'éloigner la proximité politique dans la gestion opérationnelle. Pour favoriser des investissements adaptés aux réalités du terrain, il est nécessaire selon eux de proposer un nouveau modèle de gestion financière. Ces suggestions soutiennent également les recommandations mises en avant par la Commission d'enquête sur les relations entre les Autochtones et certains services publics (2019).

Les participants proposent également de revoir certains aspects propres à la structure des corps de police autochtones. Pour surmonter la stigmatisation et favoriser la reconnaissance de leur rôle auprès des citoyens, mais plus largement au sein de la communauté policière, ils suggèrent d'attribuer un niveau de service aux corps de police autochtones. La légitimation du travail policier en contexte autochtone est, selon les participants, une cible d'action à prioriser. De plus, une régionalisation de certains corps de police autochtones pourrait être envisagée afin d'offrir une mobilité au personnel pour contrer la stigmatisation dont ils se disent victimes.

Du point de vue opérationnel, il est proposé d'améliorer la concertation entre les services policiers autochtones et les services de soutien afin d'intervenir efficacement auprès des citoyens en situation de vulnérabilité. Une revue systématique menée par l'équipe de LopezCarmen et al. (2019) suggère que les interventions intersectorielles contribuent à une amélioration de la santé mentale des enfants autochtones, mais aussi celle de leurs familles et de la communauté plus largement. Pour répondre à cette recommandation, il est proposé de formaliser des initiatives de concertation multidisciplinaires qui s'inscrivent dans le cadre de pratiques orientées vers le client. Ce type d'approche vise à trouver la meilleure solution pour répondre aux besoins des clientèles, qu'elle soit axée vers le punitif ou le curatif. Dans 
le domaine de la santé, ces pratiques de prise de décision multidisciplinaires montrent des résultats fort encourageants (Tremblay et al., 2011). Les policiers en milieux autochtones peinent également à surmonter le déficit de légitimité dont ils souffrent, entre autres, car ils ont l'obligation d'appliquer un droit étatique qui ne correspond que très partiellement aux réalités et aux besoins des collectivités autochtones (Jaccoud et Spielvogel, 2018). Ceci est particulièrement vrai lorsqu'il est question d'intervenir auprès d'enfants dont la sécurité est compromise (Bellot et Sylvestre, 2016). Il pourrait être intéressant de créer un espace de dialogue entre les corps de police autochtones, les services sociaux en protection de l'enfance, et les citoyens autochtones afin de formaliser des façons de faire respectant les spécificités des besoins de chacun. Ce constat rejoint également les recommandations proposées par Jaccoud et Spielvogel (2018) indiquant qu'il faut adapter les programmes existants et en construire d'autres à l'aide de nouvelles stratégies policières en matière de résolution de problèmes.

Finalement, la formation demeure un enjeu important pour les corps de police autochtones en raison des contraintes budgétaires, mais aussi en raison de la surreprésentation des problèmes sociaux au sein des communautés. Comme il a été expliqué,

les services de police autochtones disposent, en principe, des mêmes pouvoirs et prérogatives que les organisations policières régulières et semblent soumis à des standards d'efficacité équivalents, les moyens dont elles (sic) bénéficient leur demeurent très inférieurs, alors qu'elles doivent gérer des problématiques plus lourdes. (Jaccoud et Spielvogel, 2018, p. 36)

Dans ce contexte, il pourrait être opportun de bonifier la formation initiale et continue des policiers appelés à intervenir en contextes autochtones vers une formule axée sur les enjeux connus de détresse psychosociale, d'itinérance, de vulnérabilité, de violence et de dépendance aux substances des communautés (Bellot et Sylvestre, 2016; Kiedrowsky et al., 2017). Il importe de fournir aux policiers les outils pour intervenir dans des contextes difficiles (Griffiths et Clark, 2017), notamment pour les recrues qui amorcent leur carrière en milieux autochtones. L'insuffisance de la formation continue est aussi particulièrement préoccupante (Jaccoud et Spielvogel, 2018). Les participants à la MAG suggèrent de mettre en place des mécanismes d'accompagnement dans une logique de formation continue sur le terrain. Ils insistent aussi sur la nécessité de bonifier la préparation psychologique des futurs policiers, mais surtout d'instaurer des mesures de soutien en carrière 
pour diminuer l'isolement professionnel et social qu'ils vivent. Plusieurs études ont montré que l'exposition récurrente aux problèmes sociaux ainsi que le stress vécu par les policiers peuvent entraîner de la détresse psychologique (Bergman, Christopher et Bowen, 2016; Gershon, Barocas, Canton, Li et Vlahov, 2009). Il importe donc de les outiller afin d'amenuiser ce risque.

\section{Conclusion}

Cette étude exploratoire représente le point de départ d'une démarche d'actualisation des savoirs quant aux réalités contemporaines du travail policier en milieux autochtones. En suscitant la réflexivité des participants, il a été possible de dégager les convergences et divergences au sein de leurs propos pour en développer une compréhension globale. La théorie a éclairé les réalités vécues par les participants, lesquelles ont alimenté la théorie pour développer de nouveaux savoirs. Cet aller-retour reprend les principes de l'herméneutique où la circulation des idées permet de dépasser la théorie en proposant de nouvelles perspectives (Corbin, 2012; Van Campenhoudt et al., 2005). Cette première MAG aura donc permis l'émergence d'enjeux méconnus ou la réaffirmation de défis documentés, mais elle a surtout contribué à ouvrir vers des solutions issues des policiers patrouilleurs pour y répondre. Il importe de mettre en lumière les enjeux pour construire sur de nouvelles perspectives sensibles aux besoins de l'ensemble des collectivités autochtones et des professionnels qui interviennent auprès d'elles.

Néanmoins, il importe de rappeler que les résultats de cette présente démarche sont le reflet des réalités telles que perçues par les sept participants à cette MAG, et ne sauraient se substituer à l'ensemble des points de vue sur la question. Les conclusions ne représentent donc pas nécessairement l'opinion de l'ensemble des policiers œuvrant auprès de clientèles autochtones au Québec. Il est probable que plusieurs autres représentations puissent venir nuancer l'état actuel des réalités du travail policier en milieux autochtones. Les travaux amorcés dans le cadre de la présente démarche se poursuivront auprès d'autres policiers œuvrant auprès de clientèles autochtones, dont ceux dans le nord du Québec ou encore en centres urbains. Étant donné la diversité de ces réalités, un regard complémentaire s'impose. 


\section{Références}

Aguiar, W. et Halseth, R. (2015). Aboriginal peoples and historic trauma: The processes of intergenerational transmission. Prince George, ColombieBritannique: National Collaborating Centre for Aboriginal Health.

Aubert, L. et Jaccoud, M. (2009). Genèse et développement des polices autochtones au Québec: sur la voie de l'autodétermination. Criminologie, 42(2), 101-119.

Aubert, L. et Jaccoud, M. (2012). La Politique sur la Police des Premières Nations: une avancée en matière de gouvernance? Canadian Journal of Criminology or Criminal Justice, 54(3), 265-285.

Bellot, C. et Sylvestre, M.-E. (2016). La judiciarisation de l'itinérance à Val-d'Or. Montréal, Québec: Université de Montréal.

Bergman, A. L., Christopher, M. S. et Bowen, S. (2016). Changes in facets of mindfulness predict stress and anger outcomes for police officers. Mindfulness, 7(4), 851-858.

Brassard, R., Spielvogel, M. et Montigny, L. (2016). Réponse pénale à la violence conjugale/familiale d'hommes autochtones au Québec: quelques enjeux entourant l'intervention policière et l'incarcération. Société de criminologie du Québec, 37-44.

Brzozowski, J. A., Taylor-Butts, A. et Johnson, S. (2006). Victimization and offending among the Aboriginal population in Canada. Ottawa, Ontario: Canadian Centre for Justice Statistics.

Canadian Association of Police Governance. (2016). Governance of Policing and First Nations Communities: CAPG Conference Report 2016. Ottawa, Ontario: Canadian Association of Police Governance.

Cao, L. (2014). Aboriginal people and confidence in the police. Canadian Journal of Criminology and Criminal Justice, 56(5), 499-526.

Chartrand, L. et Horn, K. (2016). Un rapport sur les relations entre la justice réparatrice et les traditions juridiques autochtones au Canada. Ministère de la Justice Canada.

Chartrand, V. (2019). Unsettled times: Indigenous incarceration and the links between Colonialism and the Penitentiary in Canada. Canadian Journal of Criminology and Criminal Justice, 61(3), 67-89.

Chrismas, R. (2012). The people are the police: Building trust with Aboriginal communities in contemporary Canadian society. Canadian Public Administration, 55(3), 451-470.

Commission d'enquête sur les relations entre les Autochtones et certains services publics au Québec: écoute, réconciliation et progrès. (2019). Rapport final. Repéré à https://www.cerp.gouv.qc.ca/

Commission de la santé et des services sociaux des Premières Nations du Québec et du Labrador. (2018). Enquête régionale sur la santé des Premières Nations du Québec. Repéré à http://www.cssspnql.com/champs-intervention/ secteur-recherche/enquetes-populationnelles/enquete-regionale-sante

Corbin, J. (2012). Préface. Dans J. Luckerhoff et F. Guillemette (dir.), Méthodologie de la théorisation enracinée (p. VII). Québec, Québec: Presses de 1'Université du Québec. 
Enquête nationale sur les femmes et les filles autochtones disparues et assassinées. (2017). Réclamer notre pouvoir et notre place: le rapport final de l'Enquête nationale sur les femmes et les filles autochtones disparues et assassinées. Repéré à https://www.mmiwg-ffada.ca/fr/final-report/

Finkler, H. W. (1980). Les Inuit et l'administration de la justice: le cas de Frobisher Bay (T. N.-O.). Éditions Hurtubise HMH.

Fitzgerald, R. T. et Carrington, P. J. (2008). The neighbourhood context of urban Aboriginal crime. Canadian Journal of Criminology and Criminal Justice, 50(5), 523-557.

Gershon, R. R., Barocas, B., Canton, A. N., Li, X. et Vlahov, D. (2009). Mental, physical, and behavioural outcomes associated with perceived work stress in police officers. Criminal Justice and Behaviour, 36(3), 275-289.

Glaser, B. et Strauss, A. (1967). The discovery of grounded theory: Strategies for qualitative research. Chicago, IL: Aldine Publishing.

Griffiths, C. T. et Clark, P. (2017). Building police legitimacy in a high demand environment: The case of Yukon, Canada. Policing: An International Journal, 40(3), 560-573.

Griffiths, C. T. et Yerbury, J. C. (1984). Natives and criminal justice policy: The case of native policing. Canadian J. Criminology, 26(2), 147-160.

Guillemette, F. et Lapointe, J.-R. (2012). Illustration d'un effort pour demeurer fidèle à la spécificité de la méthodologie de la théorisation enracinée. Dans J. Luckerhoff et F. Guillemette (dir.), Méthodologie de la théorisation enracinée: fondements, procédures et usages (p. 11-35). Québec, Québec: Presses de 1'Université du Québec.

Jaccoud, M. (1999). Les cercles de guérison et les cercles de sentence autochtones au Canada. Criminologie, 32(1), 7-105.

Jaccoud, M. et Spielvogel, M. (2018). Les services policiers en contexte autochtone: une recension des écrits. Commission d'enquête sur les relations entre les Autochtones et certains services publics au Québec: écoute, réconciliation et progrès.

Jones, N. A., Mills, R. G., Ruddell, R. et Quinn, K. (2016). Policing First Nations. Community perspectives. Régina, Saskatchewan: Collaborative Centre for Justice and Safety.

Jones, N. A., Ruddell, R. et Summerfield, T. (2019). Community policing: Perceptions of officers policing Indigenous communities. Canadian Journal of Criminology and Criminal Justice, 61(1), 41-65.

Kiedrowski, J. S., Petrunik, M. et Ruddell, R. (2016). Illustrative case studies of First Nations policing program models. Ottawa, Ontario: Public Safety Canada.

Kiedrowski, J., Jones, N. A. et Ruddell, R. (2017). 'Set up to fail? 'An analysis of self-administered indigenous police services in Canada. Police Practice and Research, 18(6), 584-598.

Konaté, B., Berthé, A., Hien, H., Tou, F., Millogo, A., Badini-Kinda, F. et Franssen, A. (2019). Isolement résidentiel et cohabitation intergénérationnelle au Burkina Faso. Gérontologie et société, 41(1), 139-151.

La Prairie, C. (2002). Aboriginal over-representation in the criminal justice system: A tale of nine cities. Canadian J. Criminology, 44(2), 181-208. 
LeBeuf, M. E. (2011). The role of the Royal Canadian Mounted Police during the Indian residential school system. Ottawa, Ontario: Royal Canadian Mounted Police.

Lithopoulos, S. et Ruddell, R. (2011). Policing isolated Aboriginal communities: Perspectives of Canadian officers. Policing: An international journal of police strategies and management, 34(3), 434-453.

Lithopoulos, S. et Ruddell, R. (2013). Aboriginal policing in rural Canada: Establishing a research agenda. International journal of rural criminology, 2(1), 101-125.

Lopez-Carmen, V., McCalman, J., Benveniste, T., Askew, D., Spurling, G., Langham, E. et Bainbridge, R. (2019). Working together to improve the mental health of indigenous children: A systematic review. Children and Youth Services Review, 104, 1-11.

McDonough, B. (2013). Le drame des pensionnats autochtones. Relations, (768), 33-35

Ministère de la Sécurité publique. (2017). Corps de police autochtones. Repéré à http://www.securitepublique.gouv.qc.ca/police/quebec/services-depolice/police-autochtone.html

Ministère de la Sécurité publique. (2020). Les six niveaux de services policiers en fonction du nombre d' habitants. Repéré à https://www.securitepublique.gouv. qc.ca/police/quebec/services-de-police/desserte-policiere/six-niveauxservice.html

Ministère des Affaires indiennes et du Nord canadien. (1990). Rapport $d u$ maintien de l'ordre dans les réserves indiennes. Ottawa, Ontario: Canada. Ministère des Affaires indiennes et du Nord canadien.

Monchalin, L. (2016). The colonial problem: An indigenous perspective on crime and injustice in Canada. Toronto, Ontario: University of Toronto Press.

Mucchielli, A. et Paillé, P. (2003). L'analyse qualitative en sciences bumaines et sociales. Paris, France: Armand Colin.

Posca, J. (2018). Portrait des inégalités socioéconomiques touchant les Autochtones au Québec. Institut de recherche et d'informations socioéconomiques.

Roberts, J. V. et Reid, A. A. (2017). Aboriginal incarceration in Canada since 1978: Every picture tells the same story. Canadian Journal of Criminology and Criminal Justice, 59(3), 313-345.

Ruddell, R. et Jones, N. A. (2018). Policing the 'middle of nowhere': Officer working strategies in isolated communities. Policing: A Journal of Policy and Practice, 14(2), 414-427.

Ruddell, R. et Lithopoulos, S. (2013). Aboriginal policing in rural Canada: Establishing a research agenda. International Journal of Rural Criminology, 2(1), 101-125.

Salée, D. (2005). Peuples autochtones, racisme et pouvoir d'État en contextes canadien et québécois: Éléments pour une réanalyse. Nouvelles pratiques sociales, $17(2)$, 54-74.

Sécurité publique Canada. (2016). Modèles du Programme des services de police des Premières Nations - Études de cas représentatives. Repéré à https://www.securitepublique.gc.ca/cnt/rsrcs/pblctns/2016-r014/index-fr.aspx 
Statistique Canada. (2017). Série «Perspective géographique», Recensement de 2016 (Produit no 98-404- X2016001 au catalogue de Statistique Canada). Ottawa, Ontario.

Tremblay, D., Roberge, D., Cazale, L., Touati, N., Maunsell, E., Latreille, J. et Lemaire, J. (2011). Evaluation of the impact of interdisciplinarity in cancer care. BMC health services research, 11(1), 144.

Tulloch, M. H. (2017). Report of the Independent Police Oversight Review. Ministry of the Attorney General.

Van Campenhoudt, L., Chaumont, J. M. et Franssen, A. (2005). La méthode d'analyse en groupe. Paris, France: Dunod.

\section{Policing issues in Indigenous communities of Quebec. The group analysis method in the search for solutions}

ABSTRACT - Although some authors have studied Indigenous policing, very few, if any, have examined Indigenous policing from a comprehensive and qualitative approach and from the perspective of police officers. Adopting a participative method, this study used the group analysis method with seven police officers from Indigenous police forces in Quebec to document the main policing issues and formulate possible solutions adapted to the realities of the community. The results highlight, among other things, issues related to the non-recognition of the role of police within the Indigenous and even the police community, citizen and political proximity, social and professional isolation, and the lack of organizational support, as well as the lack of accessibility of partner services to support their interventions. These challenges are juxtaposed with the important social problems in the communities that police officers encounter daily. This approach gives rise to possible solutions based on the variety of representations and also led to the development of a research project aimed at gaining a better understanding of the perception of police officers who work with First Nations, Métis, or Inuit communities.

KEYWORDS • Group analysis, policing, indigenous communities, issues.

\section{Asuntos del trabajo policial en las comunidades indígenas en Quebec. El método de análisis de grupo al servicio de la búsqueda de soluciones}

RESUMEN - A pesar de que varios autores se han interesado por la policía indígena, muy pocos, o ninguno, se han interesado desde una aproximación comprensiva y cualitativa, desde el punto de vista de los policías. Adoptando una perspectiva participativa, este proyecto busca experimentar el método de análisis de grupo con siete policias de diferentes fuerzas de policía indígena quebequense para documentar los dilemas principales del trabajo de los policías en medios autóctonos y formular pistas de solución adaptadas a las realidades de las comunidades. Los resultados evidencian dilemas ligados al no reconocimiento de su rol en la comunidad indigena y policial, a la falta de apoyo organizacional, a la proximidad ciudadana y política, al aislamiento 
profesional, así como al acceso insuficiente de los servicios de los socios en apoyo a sus intervenciones. Estos desafios se yuxtaponen a las problemáticas sociales importantes enfrentadas por estos policías en su vida diaria. Este método lleva a proponer pistas de solución sustentadas en la diversidad de representaciones, las cuales permitieron, igualmente, la elaboración de un proyecto de investigación que buscaba profundizar la comprensión de la percepción de los policías que intervienen con las Primeras Naciones, los Mestizos, o los Inuit.

PALABRAS CLAVE - Método de análisis de grupo, intervención policial, indígenas, dilemas. 